\title{
IV Eigentumsrechtliche Bewertung am Beispiel der Mietpreisregulierung
}

Angesichts der Vielzahl der Instrumente sowie der divergierenden Komplexität und Klarheit ihrer ökonomischen Bewertung soll im Weiteren eine Fokussierung auf die unmittelbare Mietpreisregulierung erfolgen. Ausgehend von einer kritischen Analyse des Beschlusses des BVerfG zur „Mietpreisbremse“ im BGB (1.) soll der Berliner „Mietendeckel“ (2.) beleuchtet werden.

\section{1 „Mietpreisbremse“ des BGB im Lichte des Beschlusses des Bundesverfassungsgerichts}

\section{a) Der Beschluss des Bundesverfassungsgerichts}

Das BVerfG hat in seinem Beschluss vom 18. Juli $2019^{71}$ die angegriffenen Kernregelungen der „Mietpreisbremse“ im BGB als grundrechtskonform bewertet. Die Regulierung der Miethöhe bei Neuvermietungen nach $\S 556 \mathrm{~d}$ Abs. 1 BGB als Herzstück der „Mietpreisbremse“ hat das Gericht dabei am Maßstab der Eigentumsfreiheit aus Art. 14 Abs.1 S. 1 GG sowie der Vertragsfreiheit aus Art. 2 Abs. 1 GG und des allgemeinen Gleichheitssatzes aus Art. 3 Abs. 1 GG geprüft. Hier soll zunächst vor allem die Argumentation zur Verhältnismäßigkeitsprüfung im Rahmen der Anwendung der Eigentumsfreiheit kritisch beleuchtet werden.

\section{b) Die Identifikation des legitimen Ziels}

Basierend auf der ständigen und auch überzeugenden Rechtsprechung des BVerfG zur erhöhten Sozialbindung des Wohneigentums und des damit einhergehenden erweiterten Spielraums des Gesetzgebers bei der Schaffung von Inhaltsund Schrankenbestimmungen wirft schon die Analyse des legitimen Ziels Fragen auf. So dekretiert das Gericht: „Der gesetzgeberische Zweck, durch die Begrenzung der Miethöhe bei Wiedervermietung der direkten oder indirekten Verdrängung wirtschaftlich weniger leistungsfähiger Bevölkerungsgruppen aus stark

71 BVerfG, Beschl.v. 18.7.2019-1 BvL 1/18, abrufbar im WWW unter der URL http://www.bverfg. de/e/lk20190718_1bvl000118.html - ECLI:DE:BVerfG:2019:1k20190718.1bvl000118 (zuletzt abgerufen am 1.7.2020).

Ә OpenAccess. ( 2020 Juergen Kuehling, publiziert von De Gruyter. (cc) BY-NC-ND Dieses Werk ist lizenziert unter der Creative Commons Attribution-NonCommercial-NoDerivatives 4.0 License. 
nachgefragten Wohnquartieren entgegenzuwirken, liegt im öffentlichen Interesse. “72 Warum? Ist dies ein Selbstzweck? Ist ein Gebiet mit einem größeren Anteil an zahlungskräftigen Bewohnerinnen und Bewohnern weniger gut als ein solches mit einem geringeren Anteil? Wenn ja, warum? Ist die Wahrung einer diesbezüglich größeren Heterogenität damit ein Selbstzweck oder nur ein Zwischenziel auf dem Weg zum möglicherweise sehr mittelbar damit verknüpften Zweck der Förderung der Kreativität (was eher abwegig erscheint ${ }^{73}$ ) oder (vielleicht noch plausibler) des sehr mittelbar bekämpften sozialen Abstiegs von Wegzugsgebieten? Und ist nur die Veränderung, so klingt die Aussage, negativ und damit die Bekämpfung der Veränderung im öffentlichen Interesse? Ist also ein bereits etabliertes Viertel für Besserverdiener zu akzeptieren, nicht aber die Änderung eines bislang durchwachsenen Wohnquartiers hin zu einem solchen Viertel? Und wenn ja, warum? Geht es nicht vor allem darum, ein politisch nachvollziehbares „Recht auf Heimat“ zu schützen, das sich bei steigenden Mietpreisen einkommensschwächere Haushalte weniger gut sichern können als einkommensstärkere Haushalte, mit den damit allerdings einhergehenden, bereits oben aufgezeigten Schwierigkeiten der Präzisierung (siehe oben II.2.b))?

\section{c) Die Prüfung der Geeignetheit}

Bei der Prüfung der Geeignetheit lässt das BVerfG in ständiger Rechtsprechung die Möglichkeit der Zweckerreichung genügen. Hier startet das BVerfG seine Analyse mit einem realistischen Blick auf die begrenzte Wirkkraft der „Mietpreisbremse“ ${ }^{74}$ Sie dürfte dazu führen, dass die Nachfrage angesichts der gekappten Preise steige und damit weitere Parameter wie die Bonität der potenziellen Mieter bei der Vergabe der Wohnung noch stärker in den Vordergrund rückten und das Ziel eines breiteren Zugangs für weniger zahlungskräftige Mietsuchende gar nicht erreicht werde. ${ }^{75}$ Auch dass die Regelung eine Verknappung des Angebots und damit eine weitere Verschärfung der Situation bedinge, sei nicht ausgeschlossen. Diesen naheliegenden negativen Effekten stellt das BVerfG eine große Unsicherheit hinsichtlich des gewünschten positiven Effekts gegenüber: So sei die Möglichkeit, dass einkommensschwächere Nachfrager bei einem Wohnungswechsel

72 Ebenda, Rn. 59.

73 Siehe aber den Nachweis in Fn. 49.

74 BVerfG, Beschl.v. 18.7.2019-1 BvL 1/18, Rn. 62, abrufbar im WWW unter der URL http://www. bverfg.de/e/lk20190718_1bvl000118.html - ECLI:DE: BVerfG:2019:1k20190718.1bvl000118 (zuletzt abgerufen am 1.7.2020); vgl. auch Rn. 65.

75 Ebenda, Rn. 62 und 64. 
in ihrem Wohnquartier aufgrund des niedrigeren Mietniveaus eine neue Bleibe finden, lediglich „nicht auszuschließen“. ${ }^{76}$

\section{d) Erforderlichkeitsprüfung}

Im Rahmen der Erforderlichkeitsprüfung schärft das Gericht die Anforderungen an alternative Handlungsinstrumente und nicht an das $\mathrm{zu}$ prüfende grundrechtsbeeinträchtigende Instrument. Alternativen müssen in ihrer gleichen Wirksamkeit „zweifelsfrei“ sein. ${ }^{77}$ Der Gesetzgeber müsse hier nicht „die beste Lösung“ wählen und ihm sei insoweit ein „Beurteilungs- und Prognosespielraum“ zuzubilligen. Diese strengen Maßstäbe sind mit Blick auf die Wahrung des legislativen Gestaltungsspielraums noch nachvollziehbar. Der nächste Argumentationsschritt ist dann aber fraglich: Das BVerfG attestiert zwar die breite Streuwirkung der Regelung, die allen Wohnungssuchenden zugutekommt und erkennt auch die alternativen Instrumente wie „die Förderung des Wohnungsbaus“ und die Stärkung der Nachfrager durch die Erhöhung des Wohngelds. Nach unklarem Hinweis auf die „mit diesen Maßnahmen verbundenen Kosten“ („Ungeachtet“), sei aber schlichtweg „nicht erkennbar, dass der Gesetzgeber diese im Rahmen seines Prognose- und Beurteilungsspielraums als gegenüber der Miethöhenregulierung mildere und zweifelsfrei - auch kurzfristig - vergleichbar wirksame Mittel hätte heranziehen müssen.“78

Der Maßstab „nicht erkennbar“ ist insoweit unbefriedigend. Unklar ist auch, worauf sich der „Prognose- und Beurteilungsspielraum“ genau bezieht. Hinsichtlich der Kosten dürfte es eher Aufgabe des BVerfG sein, die Kostenübernahme grundrechtlich $\mathrm{zu}$ bewerten. Einmal das Ziel unterstellt, dass einkommensschwächere Nachfrager leichter Zugang zu bestimmten Wohngegenden erhalten und „Wohnquartiersangehörige“ als „Insider“ im Falle des Wohnungswechsels leichter im Quartier bleiben können und diese Maßnahme Geld kostet, da sie nur über den Preis einer Kappung der Mietpreise möglich ist, bleibt doch die verfassungsrechtlich zu bewertende Frage: Fällt diese Aufgabe in die Verantwortung des Staates und damit der Allgemeinheit der Steuerzahler oder in die besondere soziale Verantwortung des Eigentümers bzw. muss dieser entsprechende Lasten akzeptieren? Im ersteren Fall sind die Kosten selbstverständlich vom Steuerzahler zu tragen. Im zweiten Fall können sie auf die Eigentümer abgewälzt werden.

76 Ebenda, Rn. 64.

77 Ebenda, Rn. 66.

78 Ebenda, Rn. 67. 
Das bringt sodann die Zielerreichungsqualität der „Gießkannenmethode“ auf den Prüfstand. Das BVerfG räumt selbst ein, dass die gewünschte Wirkung des Zugangs von einkommensschwächeren Bevölkerungsgruppen mit dem Instrument kaum zu erreichen und lediglich „nicht ausgeschlossen“79 sei. Um die Wirkung auf den Punkt zu bringen: Viele einzelne Neumieter, die das Glück eines Zugriffs auf die marktunüblich niedrigpreisigen Wohnungen haben, werden von der Kappung profitieren. Es ist unbekannt, ob und in welchem Umfang in dieser „faktischen“ Adressatengruppe die „normative“ Zielgruppe der einkommensschwachen Bevölkerung vertreten sein wird. Ökonomische Untersuchungen deuten darauf hin, dass dieser Anteil eher gering und dass die Zielgruppe eher negativ durch die Maßnahme betroffen sein wird. ${ }^{80}$ Es profitiert also weit überwiegend eine nicht schützenswerte Gruppe, während die schützenswerte Gruppe kaum bis gar nicht profitiert oder sogar Nachteile davonträgt. Wenn nun aber gerade diese Gruppe der einkommensschwachen Mieter schutzwürdig ist, warum ist dann nicht eine Maßnahme notwendig und vorzugswürdig, die gerade dieser Gruppe hilft? Dies würde allerdings voraussetzen, dass ein spezifisches Instrument entwickelt und angewandt wird, das gerade einkommensschwachen Bevölkerungsgruppen den Zugang zu jenen Vierteln eröffnet. Etabliert ist insoweit insbesondere eine besondere Zuzahlung (sogenanntes „Wohngeld“), die an einen Einkommensnachweis geknüpft und von der öffentlichen Hand getragen wird. Das macht auch die Zahlungsflüsse transparenter und verschärft die Effizienzdiskussion. Substituieren wir in einem Gedankenexperiment einmal die Maßnahme durch einen expliziten Zahlungsstrom: Jeder, der neu in ein Gebiet mit angespannten Wohnungsmärkten zieht, bekommt eine staatliche Transferzahlung in Form eines Mietenzuschusses. Erstens würde sofort deutlich, dass dies die Nachfrage in angespannten Lagen selbstverständlich verschärft, da durch die Transferzahlung mehr potenzielle Mieter als Nachfrager auftreten. Ließe sich diese Maßnahme aber, die aus der Mietersicht einen ähnlichen Effekt hätte wie die „Mietpreisbremse“ und nur die Lasten nicht auf die Vermieter mit Mietobjekten in den betroffenen Gebieten verlagert, sondern auf den Steuerzahler, politisch durchhalten? Wohl kaum. Käme dann noch heraus, dass die Beantragung des Zuschusses so komplex ist, dass er gerade viel seltener von einkommensschwachen als von einkommensstarken Haushalten in Anspruch genommen wird, wäre das Ende der Durchsetzbarkeit der Maßnahme endgültig besiegelt. Da die Kosten

79 Ebenda, Rn. 64.

80 Vandrei Preisregulierung in der sozialen Wohnungspolitik - die schädliche Wirkung der Mietpreisbremse, in: ifo Schnelldienst 21/2018, S. 18f., abrufbar im WWW unter der URL https:// www.cesifo.org/DocDL/sd-2018-21-11-8.pdf (zuletzt abgerufen am 1.7.2020); Voigtländer Luxusgut Wohnen. Warum unsere Städte immer teurer werden und was jetzt zu tun ist, 2017, S. $174 \mathrm{f}$. 
der vorliegenden Umverteilung jedoch auf die Vermieter und nicht auf den Steuerzahler verlagert werden, bleibt ein Aufschrei bei einer derartig ungenauen Steuerungsmaßnahme aus.

Damit steht fest, dass wesentlich geeignetere Maßnahmen vorhanden sind, die zugleich gar nicht in Grundrechte eingreifen. Auch auf der Zeitschiene dürfte eine Modifikation des Wohngeldes dahingehend, dass spezifische Zuschläge für die Verfolgung der genauen Ziele gezahlt werden, namentlich einkommensschwachen Personen den Zuzug zu begehrten Wohnlagen erschließen, zu bevorzugen sein. Dies würde nämlich ,auch kurzfristig“ im Sinne der Rechtsprechung des BVerfG erfolgen können. Jene Vorgehensweise würde jedoch zugleich verlangen, dass die Ziele klarer benannt und transparenter gemacht würden. Angesichts des besonders geschützten haushaltspolitischen Spielraums des Gesetzgebers ist es aber dessen Entscheidung, innerhalb des rechtlich Zulässigen besondere Gruppen spezifischen Belastungen $\mathrm{zu}$ unterwerfen und nicht den allgemeinen Steuerzahler in die Verantwortung $\mathrm{zu}$ nehmen. Relativierend ist wiederum darauf hinzuweisen, dass diese Betrachtung nur auf die unmittelbaren Effekte abstellt. Denn die „Mietpreisbremse“, der „Mietendeckel“, die Kappungsgrenze etc. belasten ebenfalls den Haushalt, da steuerpflichtige Einnahmen beim Vermieter wegfallen, die Subvention des Mieters jedoch steuerfrei ist. Zudem sind die o.a. Maßnahmen marktverzerrend. Vom Wohngeld verspricht man sich dagegen eine zielgerechtere Förderung nur der wirklich Bedürftigen und dies auch nur für die Dauer der Bedürftigkeit. ${ }^{81}$ Die angeführten negativen haushalterischen Effekte von „Mietpreisbremse“, „Mietendeckel“, Kappungsgrenze etc. sind jedoch einerseits nur mittelbar und betreffen andererseits teilweise auch gebietsfremde Haushalte, wenn etwa der Vermieter einer Wohnung in Berlin in Regensburg lebt und dort die verringerten Steuereinnahmen entstehen. So erklärt sich auch in haushalterischer Sicht die politische Popularität entsprechender Maßnahmen, da die Nutznießer lokale Mieter und damit grundsätzlich Wähler sind, während die Belasteten teils gebietsfremde Vermieter, Zuzugswillige etc. sind. Gleichwohl kann unter dem Blickwinkel der Schonung der gebietseigenen öffentlichen Haushalte vor unmittelbaren Belastungen, wenn dies ein weiteres zentrales Motiv der Instrumentenwahl sein sollte, die Maßnahme der Modifikation des Wohngeldes nicht als gleich geeignet angesehen werden. Dann könnte die Erforderlichkeit der „Mietpreisbremse“ als Maßnahme trotz ihrer geringen

81 Siehe dazu sehr ausführlich $B B S R$, bearbeitet von Voigtländer/Clamor/Henger/Niehues, Bestandsaufnahme und Wirkungsanalyse des Wohngeldes, 2015, abrufbar im WWW unter der URL https://www.bbsr.bund.de/BBSR/DE/veroeffentlichungen/sonderveroeffentlichungen/2015/DL Wohngeld.pdf?_blob=publicationFile\&v=1 (zuletzt abgerufen am 1.7.2020). 
Zielerreichung bejaht werden. Die angeführten Argumente sind jedoch im Rahmen der Abwägung von Bedeutung.

\section{e) Die Zumutbarkeitskontrolle}

Die anschließende Prüfung der Verhältnismäßigkeit im engeren Sinne durch das BVerfG wirft die Frage auf, ob der Kontrollansatz nicht zu kurz greift: So wird die Schwere des Eingriffs abgewogen gegen das Gewicht und die Dringlichkeit der Rechtfertigungsgründe, ohne dass in die Abwägung einbezogen wird, wie groß der Beitrag des Instruments zur Zielerreichung ist. ${ }^{82}$ Auch die Zielbenennung wird hier nicht präzisiert (,Aber auch in nachgefragten Stadtvierteln sind große Teile der Bevölkerung auf Mietwohnungen unausweichlich angewiesen.“; „gesellschaftspolitische Interesse an einer durchmischten Wohnbevölkerung in innerstädtischen Stadtvierteln“; „Verhinderung der Gentrifizierung“833). Wie hinderlich diese Unklarheit ist, wird in der Entscheidung auch daran deutlich, dass diese Zieldefinition in offensichtlichem Widerspruch zu der Argumentation des BVerfG steht, die zur Rechtfertigung der Berliner Mietenbegrenzungsverordnung angeführt wird. Denn hier wird der Argumentation des Berliner Senats gefolgt, dass eine Verordnung für das gesamte Berliner Stadtgebiet gerechtfertigt sei, da wegen der „polyzentrischen Ausrichtung“ Berlins dieser „ein einheitlicher Wohnungsmarkt sei, der sich territorial nicht weiter aufspalten lasse“ ${ }^{84}$ Würde dies zutreffen, unterläge ganz Berlin einem Gentrifizierungsprozess. Damit geht es gleichsam gar nicht mehr um den Ausgleich zwischen nachgefragten und nicht nachgefragten Stadtteilen Berlins, sondern um jeglichen Zuzug zum Berliner Stadtgebiet als Gentrifizierungsproblem.

Fest stehe jedenfalls, dass kein schutzwürdiges Interesse des Vermieters auf „höchstmögliche Mieteinkünfte“ 85 besteht. Als Grenzen führt das BVerfG stattdessen im Anschluss an seine bisherige Rechtsprechung ,den dauernden Verlust für den Vermieter“ und die „Substanzgefährdung der Mietsache“86 an, die allerdings vorliegend „nicht ersichtlich“ ${ }^{\text {“87 }}$ seien. Sodann erfolgt wiederum der Verweis

82 BVerfG, Beschl.v. 18.7.2019-1 BvL 1/18, Rn. 68 abrufbar im WWW unter der URL http://www. bverfg.de/e/lk20190718_1bvl000118.html - ECLI:DE:BVerfG:2019:1k20190718.1bvl000118 (zuletzt abgerufen am 1.7.2020).

83 Ebenda, Rn. 71 bzw. 72.

84 Ebenda, Rn. 116.

85 Ebenda, Rn. 76; 88.

86 Ebenda, Rn. 69; 88.

87 Ebenda, Rn. 89. 
auf den „weiten Gestaltungsspielraum“ des Gesetzgebers, ohne dass hier klarer würde, hinsichtlich welcher Parameter dieser greift. ${ }^{88}$

Im Übrigen verweist das BVerfG auf die verfahrensrechtlichen Anforderungen, die eine sorgfältige Prüfung vor Erlass einer Verordnung im jeweiligen Bundesland verlangen. Insoweit liegt dieses Anforderungsprofil durchaus auf der Linie der bisherigen zivilgerichtlichen Rechtsprechung, die erhöhte Anforderungen an die individualisierte Begründung für den Erlass der Verordnungen stellt. ${ }^{89}$

Die weiteren Ausführungen zu den abmildernden Effekten sind aus der Perspektive des einzelnen Grundrechtsträgers wenig überzeugend. Der beschränkte räumliche Anwendungsbereich der Verordnung wird diejenigen Eigentümer wenig trösten, deren ausschließliches Wohnungseigentum in diese Gebiete fällt. Sodann leidet die Prüfung daran, dass für die Gegengewichte auf das abstrakte Gewicht der Rechtfertigungsgründe abgestellt wird, nicht jedoch auf das Produkt aus der Gewichtigkeit jener Gründe und den durch die Maßnahme bei der Zielverfolgung tatsächlich bzw. vermutlich erreichten Effekten.

Zielführender sind dann schon die weiteren Überlegungen, die darauf abstellen, inwiefern sich die Miete „von der am unregulierten Markt erzielbaren Miete“ durch die Regulierungsmaßnahme „entkoppelt““.90 Hier räumt das BVerfG durch die „Mietpreisbremse“ einen weiteren Schub der Entkoppelung ein, sieht diesen aber durch den zeitlichen Verzug, die Möglichkeit eines 10\%igen Aufschlags, den begrenzten Anwendungsbereich auf ältere Bestandsbauten (älter als 1. Oktober 2014) sowie die fünfjährige Befristung als hinreichend abgemildert an. ${ }^{91}$ Damit können als verfassungsrechtliche Grenzen für weiter gehende Regulierungsinstrumente ausgemacht werden: fehlende Aufschläge bzw. solche, die nicht wenigstens die Inflation bzw. Kostensteigerungen kompensieren ${ }^{92}$, die überzogene Ausdehnung der regulierten Mieten sowie die Entfristung bzw. die zu lange Anwendungsdauer. ${ }^{93}$

88 Ebenda, Rn. 74.

89 Vgl. dazu Kühling/Drechsler Anm. zu AG München, Urt. v. 21.6.2017-414 C 26570/16: Nichtigkeit der bayerischen Mieterschutzverordnung - sogenannten Mietpreisbremse, ZfIR 2017, 619; siehe ferner die Nachweise in Fn. 20.

90 BVerfG, Beschl.v. 18.7.2019-1 BvL 1/18, Rn. 83, abrufbar im WWW unter der URL http://www. bverfg.de/e/lk20190718_1bvl000118.html - ECLI:DE:BVerfG:2019:1k20190718.1bvl000118 (zuletzt abgerufen am 1.7.2020).

91 Ebenda, Rn. 85-87.

92 Siehe dazu auch ebenda, Rn. 89.

93 Siehe dazu auch unter Gleichheitsgesichtspunkten ebenda, Rn. 101. Das BVerfG hat offengelassen, ob eine Verlängerung der „Mietpreisbremse“ um weitere fünf auf einen Zeitraum von dann zehn Jahren, wie jüngst vom Bundestag beschlossen, bereits zu einer verfassungswidrigen Unzumutbarkeit der Regelung führt. 


\section{f) Zwischenfazit}

Zwar zieht das BVerfG damit jedenfalls grob erkennbare, eigentumsrechtlich fundierte rote Linien für den Ausbau der Mietenregulierung: Diese werden allgemein tendenziell dann überschritten, wenn die regulatorischen Eingriffe eine allzu starke Abkoppelung vom freien Mietenmarkt generieren bzw. wenn die Abkoppelungsinstrumente allzu langandauernd eingesetzt werden. Individuell treten als Grenzen dauernde Verluste für Vermieter und Substanzgefährdungen des Mietobjekts hinzu.

Gleichwohl ist hinsichtlich der Argumentation kritisch zu hinterfragen, warum aus Sicht des BVerfG die geringe Zielerreichungskraft des Instruments im Rahmen der Abwägung offensichtlich keine Rolle spielt. Das kann unter dem Gesichtspunkt der sozialen Bindung des Eigentums im Ergebnis nicht überzeugen: So sind die Eigentümer umso weniger in die Pflicht zu nehmen, je schwächer der Zielerreichungsbeitrag eines Instrumentes ist. Plastisch ausgedrückt: Ein eingriffsintensives, kaum wirksames Instrument lässt sich nicht rechtfertigen, ein wirksames hingegen schon eher. Sodann wird auch deutlich, worin der Spielraum des Gesetzgebers liegt. Dieser hat einen Prognosespielraum, je unklarer die Wirkungszusammenhänge sind. Wenn also umstritten ist, ob die Maßnahme die gewünschten Effekte erzielt, obliegt es dem Gesetzgeber, bei Unsicherheit eine Entscheidung zu treffen. Ebenso kommt dem Gesetzgeber ein Gewichtungsspielraum hinsichtlich der Bedeutung der zu erreichenden Ziele zu. Wenn aber wie vorliegend - auf der Hand liegt, dass die Maßnahme das Ziel, einkommensschwachen Personen den Zugang zu angespannten Wohnungsmärkten zu erleichtern, nur schwach bis gar nicht, jedenfalls aber nur höchst zufällig erreicht, kann es eine scharfe Eingriffswirkung in die Eigentumsfreiheit nicht rechtfertigen. Die Eingriffsintensität ist bei den verschiedenen Eigentümern zwar höchst heterogen. Sie kann jedoch in Einzelfällen besonders scharf sein. Das ist etwa der Fall, wenn ein Objekt zu einem hohen Kaufpreis bei einer hohen Beleihung kurz vor Wirksamenwerden der Maßnahme erworben wurde, um aus den Einnahmen abzüglich der Kosten den eigenen Lebensunterhalt zu bestreiten.

Insoweit ist auch der überzeugende Hinweis, dass ein „Vertrauen, mit der Wohnung höchstmögliche Mieteinkünfte“944 zu generieren, nicht geschützt sei, nur ein erster Fingerzeig. Denn es bleibt die Frage, wie es mit dem Vertrauen aussieht, überhaupt auskömmliche Einkünfte zu erzielen.

$94 \mathrm{AaO}$, Rn. 76; 88. 


\section{2 „Mietendeckel“ in Berlin im Lichte der bisherigen Ausführungen}

\section{a) Wirkungen bezogen auf die eigentumsrechtlichen Schranken im Überblick}

Vergleicht man die Berliner Regelung mit der „Mietpreisbremse“ im BGB, so fällt zunächst hinsichtlich der Entkoppelungseffekte vom unregulierten Mietenmarkt auf, dass der unregulierte Mietenmarkt durch die Berliner Regelung insgesamt deutlich reduziert wird. Unreguliert sind künftig nur noch die Mieten für Gebäude, die nach dem Stichtag 2014 errichtet worden sind. Der Entkoppelungseffekt hinsichtlich des Anwendungsbereichs ratione materiae steigt also stark: Nunmehr werden alle Bestandsmieten und alle Neuvermietungen bezogen auf alle vor dem Jahr 2014 errichteten Gebäude erfasst, was einem Anteil von deutlich mehr als $90 \%$ der Wohnungen entsprechen dürfte. ${ }^{95}$ Die noch in der „Mietpreisbremse“ auf Bundesebene vorgesehenen weiteren Ausnahmen, insbesondere für hohe Vormieten, entfallen. Vor allem ist aber die materielle Entkoppelungswirkung (und damit auch die materielle Eingriffswirkung) massiv verschärft: Es erfolgt zum einen ein totaler Mietenstopp. Der „Sicherheits“-Zuschlag von 20\% greift nur für überhöhte Bestandsverträge. Selbst der Kostenanpassungs- und Inflationsausgleich wird gekappt auf höchstens 1,3\%. Die deutlichste Entkoppelungwirkung setzt jedoch dadurch ein, dass als Vergleichsmaßstab bei Neuvermietungen nicht mehr auf Vergleichsmieten abgestellt wird und damit keine Rückkoppelung an Marktmieten mehr erfolgt. Vielmehr wird eine statische Mietentabelle als Vergleichsmaßstab herangezogen, die preisrelevante Aspekte wie die Größenklasse der Wohnung, vor allem aber deren Lage nicht hinreichend abbildet. Damit wird letztlich ein Preis festgesetzt, der nur bedingt mit dem Marktpreis korreliert und diesem teils nahekommt, teilweise aber auch deutlich von ihm entfernt liegt, nämlich vor allem für Luxuswohnungen in besonders begehrten Lagen. Damit tritt auch der - sicherlich nicht erwünschte - Effekt ein, dass die Begünsti-

95 Im Jahr 2019 wurden bei 42.235 .402 bestehenden Wohnungen 293.002 fertiggestellt, was einer Errichtungsquote von ca. $1 \%$ entspricht, so dass lediglich der Wohnungsbestand der letzten fünf Jahre und damit von etwas mehr als 5\% von der Regulierung ausgenommen wird; vgl. die Daten aus Destatis Gebäude und Wohnungen, 2019, S. 7, abrufbar im WWW unter der URL https://www. destatis.de/DE/Themen/Gesellschaft-Umwelt/Wohnen/Publikationen/Downloads-Wohnen/fort schreibung-wohnungsbestand-pdf-5312301.pdf?_blob=publicationFile. Destatis Bauen und Wohnen, 2019, Tab. 4.5, abrufbar im WWW unter der URL https://www.destatis.de/DE/Themen/ Branchen-Unternehmen/Bauen/Publikationen/Downloads-Bautaetigkeit/baugenehmigungenbaufertigstellungen-pdf-5311101.pdf?_blob=publicationFile (jeweils zuletzt abgerufen am 20.9. 2020). 
gungswirkung des „Mietendeckels“ am oberen Ende der Mietpreise und damit tendenziell für einkommensstarke Mieter besonders groß ist. Ein dritter Entkoppelungseffekt tritt sodann ratione temporis ein: Die Dauer der „Mietpreisbremse“ im BGB von fünf Jahren ist bereits abgelaufen, wurde verlängert und wird mit der verschärften Regelung auf Landesebene nochmals um fünf Jahre ergänzt. Sie erstreckt sich damit auf zehn Jahre, was einem erheblichen Anteil an der Investitionslaufzeit für Wohnimmobilien entspricht. Die insgesamt eintretende Entkoppelungswirkung gegenüber den Marktmieten ist damit extrem verschärft.

Auch die individuellen Härten werden dadurch deutlich stärker zu Tage treten und in viel mehr Fällen dauernde Verluste für Vermieter und Substanzgefährdungen des Mietobjekts bedingen. Allerdings greift eine Härtefallklausel, die zu einem zentralen Vehikel zur Herstellung verfassungskonformer Zustände mutiert.

\section{b) Bewertung der Regelungen mit Blick auf die eigentumsrechtlichen Schranken im Detail}

Was bedeutet dies nun für die Bewertung der einzelnen Regelungsgehalte und der Gesamtregelung unter Verhältnismäßigkeitsgesichtspunkten?

Mit Blick auf die Bestandsmieten geht es um den Mieterhöhungsstopp und die Mietenabsenkung. Ersteres ist instrumentell vergleichbar mit der Kappungsgrenze, bloß dass die Mieterhöhung nicht auf 15\% in drei Jahren und damit auf 5\% p.a., sondern auf $0 \%$ bis 2021 und anschließend auf maximal 1,3\% p.a. gekappt wird. Da diese Regelung vollkommen unabhängig von den vorangegangenen Mieterhöhungen ist und sogar von der Inflation und Kostensteigerung entkoppelt wird, liegt keinerlei Marktversagensgrund vor. Der Mieter wird nicht vor einem opportunistischen Verhalten des Vermieters geschützt. Dies wird besonders deutlich am Umstand, dass auch Staffel- und Indexmieten erfasst werden. Es handelt sich also um eine rein verteilungspolitische Maßnahme, die allenfalls mit dem schwachen Argument der Internalisierung externer Effekte begründet werden kann. Die Maßnahme wird es zweifellos den Bestandsbewohnern erleichtern, in ihren Wohnungen zu bleiben. Das verteilungspolitische Ziel, dass Eigentümer von Wohnungen in begehrten Wohnlagen Mieter in begehrten Wohnlagen subventionieren, wird also erreicht. Aber dies erfolgt vollkommen unabhängig von etwaigen Bedürfnislagen. Um einen besonders plastischen Fall der abstrusen Verteilungseffekte aufzuzeigen: Im schlimmsten Fall wird der reiche Mieter einer Zweitwohnung in der begehrten Wohnlage subventioniert durch den einkommensschwachen Selbständigen im Rentenalter, der sich dort eine Wohnung gekauft hat, um die Mieteinnahmen als wesentlichen Baustein seiner Altersvorsorge 
einzusetzen. Die Härtefallklausel hilft dem Vermieter in diesem Beispiel nur sehr begrenzt und auch abhängig davon, wie sie angewandt wird.

Nicht hinreichend geklärt ist etwa, was genau unter dem Erfordernis des $\S 8$ Abs. 2 S. 1 MietenWoG Bln zu verstehen ist, dass die Miethöhe „auf Dauer zu Verlusten für die Vermieterinnen und Vermieter“ führt. Damit werden sicherlich zutreffend eindeutig verfassungswidrige Sachverhaltskonstellationen erfasst. Schon diese werden aber dadurch relativiert, dass der Vermieter nur solche Gründe anführen kann, die nach $\S 8$ Abs. 1 S. 2 MietenWoG Bln nicht in seinem Verantwortungsbereich liegen. Was damit gemeint ist, wird auch in der letzten Ergänzung im Gesetzgebungsverfahren nicht hinreichend erläutert. In der ursprünglichen Gesetzesbegründung wurde lediglich darauf hingewiesen, dass „vermieterseitig vermeidbare Umstände ausgeschlossen [sind]“996. Nunmehr spricht das Gesetz ganz allgemein von Renditeerwartungen, die vermeidbar seien, ohne dass damit klar wird, ob damit auch moderate Renditeerwartungen zur Finanzierung etwa der Altersvorsorge gemeint sind. Genauso unklar bleibt der Ausschluss von „Finanzierungskosten außerhalb des Marktüblichen“. Sind damit etwa zu hohe Ausgangsbeleihungen gemeint, die der Vermieter bei einer „solideren Finanzierung“ hätte vermeiden können? Wenn ja, wann liegen diese vor? Zwar wurde insoweit in $\S 8$ Abs. 2 S. 2 MietenWoG Bln ergänzt, dass ein Verlust anzunehmen ist, „wenn die laufenden Aufwendungen die Erträge für die maßgebliche Wirtschaftseinheit übersteigen“. Können damit, was zu vermuten ist, aber unklar bleibt, die Kapitalkosten vollumfänglich, also einschließlich der fixierten Tilgung, geltend gemacht werden? Oder kann bzw. muss hier die Genehmigungsbehörde wiederum eine marktunüblich hohe Tilgung, wann auch immer diese vorliegt, nach $\S 8$ Abs. 1 S. 2 MietenWoG Bln als im Verantwortungsbereich des Vermieters liegend annehmen und daher einen Härtefall verneinen?

Völlig unabhängig davon wird aber jedenfalls die Absicht zur Erzielung von Erträgen und damit die Versorgungsabsicht schon dem Wortlaut des Gesetzes nach nicht als Härtefall anerkannt. Stattdessen wird lediglich in der Gesetzesbegründung auf die entsprechende Notwendigkeit verwiesen, dass die gesetzliche Regelung nicht dem Vermieter die Möglichkeit verwehren darf, durch die Vermietung „den Ertrag zu ziehen, der zur finanziellen Grundlage für die eigene

96 Abgeordnetenhaus Berlin, Vorlage zur Beschlussfassung vom 28.11.2019, Gesetz zur Neuregelung gesetzlicher Vorschriften zur Mietenbegrenzung, AGH-Drs. 18/2347, S. 35; inzwischen ist auf Basis des $\S 8$ Abs. 3 MietenWoG Bln die Verordnung zur Regelung der für einen Härtefall maßgeblichen Kriterien nach dem Gesetz zur Mietenbegrenzung im Wohnungswesen in Berlin (Härtefallverordnung - HärteVO) vom 5. Juni 2020, GVBl. Berlin, S. 522, erlassen worden. 
Lebensgestaltung beiträgt“97. Wenn die Anerkennung eines entsprechenden Härtefalls sicher gewollt ist, wäre aber die Regelung im Gesetz selbst erforderlich. Hier wäre es nun gut zu wissen, in welchem Umfang Renditeziele nach Auffassung des BVerfG von der Eigentumsfreiheit geschützt sind und welcher Rechtfertigungsgründe in welcher Gewichtigkeit es bedarf, um diese Chancen regulatorisch $\mathrm{zu}$ unterdrücken.

Auch unter Vertrauensschutzgesichtspunkten ist darauf hinzuweisen, dass die gesamte Wohnungspolitik der Nachkriegsgeschichte Deutschlands und auch der Zeit nach der Wiedervereinigung von einem Interessenausgleich zwischen Vermieter und Mieter geprägt ist. Dieser Ansatz wird durch die Berliner Regelung gesprengt, da nunmehr einseitig zulasten der Vermieter eine Lösung gewählt wird. Sollte gemessen an Art. 14 GG eine Regulierung zulässig sein, die eine Gewinnerzielungsabsicht mit Immobilien faktisch ausschließen darf, ist ein Wohneigentum als Kapitalanlage betriebswirtschaftlich nicht mehr vernünftig. Die Eingriffswirkung ist dann extrem scharf. Faktisch läuft die Regelung für eine Vielzahl von Fällen aber darauf hinaus. Dasselbe gilt für die entsprechende Regelung von Neuvermietungen. Dort entfällt sogar der 20\%ige Zuschlag, der für Bestandsvermietungen noch greift. Der Vertrauensschutz auf die langfristige Rentabilität einer Kapitalanlage in Wohnimmobilien im Sinne einer auskömmlichen, keineswegs maximalen Rendite wird daher auch deutlich stärker beeinträchtigt: Es hängt letztlich vom Zufall ab, ob man einen Mieterwechsel mit dem Verlust des 20 \%igen Zuschlags hat oder nicht. Da im Unterschied zur Regelung im Bundesgesetz auch kein Schutz höherer Vorvermietungen greift, ist der Vertrauensschutz nochmals stark reduziert.

Da die Regelung insgesamt vollkommen unabhängig von der Einkommenssituation des Mieters greift, sind die Effekte hinsichtlich des verfolgten Zweckes des Schutzes einkommensschwacher Haushalte und der Steigerung deren Verbleib- und Zuzugsperspektiven extrem unsicher. Bei Bestandsmieten ist der Streuund damit auch der „Mitnahme“-Effekt extrem groß. D.h., es werden auch sehr viele Mieter einen Vorteil erlangen, die auch bei stärkeren Mietsteigerungen noch im Viertel wohnen bleiben würden. Gewiss werden aber auch einkommensschwache Haushalte davon profitieren und gegebenenfalls auch solche, die andernfalls ihr Mietobjekt wechseln müssten. Bei Neuvermietungen dürfte dieser gewünschte Effekt noch deutlich geringer sein, da die Regelung faktisch eher einkommensschwache Haushalte bei der Wohnungssuche benachteiligen dürfte.

97 AaO, S. 35 unter Hinweis auf BVerfG, Beschl. v. 18.7.2019-1 BvL 1/18, Rn. 53, abrufbar im WWW unter der URL http://www.bverfg.de/e/lk20190718_1bvl000118.html - ECLI:DE:BVerfG:2019: lk20190718.1bvl000118 (zuletzt abgerufen am 1.7.2020). 
Damit liegt durch die Bündelungswirkung der verschiedenen Vorgaben ein extrem scharfer Eingriff vor. Der Anteil unregulierter Mietverhältnisse schrumpft deutlich. Schon dies führt zu einer starken Entkoppelung vom unregulierten Mietenmarkt. Im Ergebnis kann damit schon bei einer Anwendungsdauer von nur fünf Jahren, die ja in Fortsetzung der bereits erfolgten fünfjährigen Wirkung der „Mietpreisbremse“ für einzelne Objekte greift, in zahlreichen Fällen eine Gewinnerzielungsmöglichkeit mit Mietwohnungen ausgeschlossen werden. Die Belastungswirkungen werden dabei extrem heterogen sein, je nachdem, zu welchem Zeitpunkt das Objekt mit welcher Finanzierung zu welchem Preis gekauft worden ist. Angesichts der langandauernden, mindestens zehnjährigen Gesamtwirkung der Berliner Regelung und der verschärften Abkoppelung vom freien Mietenmarkt (und den Vergleichsmieten) sind selbst die vom BVerfG weit gezogenen roten Linien im Rahmen der Verhältnismäßigkeitsprüfung überschritten. Das gilt im Rahmen der Abwägung erst recht dann, wenn die Steuerungswirkung zur Zielerreichung in die Abwägung einbezogen wird. Diese ist für Bestandsmieten mit großen Streueffekten verbunden und für Neuvermietungen eher negativ. Ferner kommen die weiteren negativen Effekte für die Wohnungssuchenden hinzu, die aufgrund der negativen Investitionsanreize auf ein verknapptes Angebot stoßen werden. Schließlich werden in einer Reihe von Fällen auch noch zusätzliche summarische Belastungseffekte der Eigentümer durch andere Regulierungsinstrumente hinzukommen. Das gilt insbesondere im Fall von Verkaufsbeschränkungen im Rahmen einer denkbaren Umwandlung der Wohnung von einer fremd- in eine selbstgenutzte Wohnung. ${ }^{98}$

98 Gemäß § 172 Abs. 1 S. 4 BauGB werden die Landesregierungen zum Erlass einer Verordnung ermächtigt, die eine Begründung von Wohnungseigentum an Gebäuden in Milieuschutzgebieten unter Genehmigungsvorbehalt stellt; vgl. dazu Stock in: Ernst/Zinkahn/Bielenberg/Krautzberger (Hrsg.), BauGB Kommentar, § 172 Rn. 115, 126. Ergänzungslieferung (Stand: August 2017). 